\title{
An Area-efficient Microstrip Diplexer with a Novel Structure and Low Group Delay for Microwave Wireless Applications
}

\author{
Salah I. Yahya ${ }^{1,2}$ and Abbas Rezaei ${ }^{3}$ \\ ${ }^{1}$ Department of Communication and Computer Engineering, Cihan University-Erbil, \\ Erbil, Kurdistan Region - F.R. Iraq \\ ${ }^{2}$ Department of Software Engineering, Faculty of Engineering, Koya University, \\ Koya KOY45, Kurdistan Region - F.R. Iraq \\ ${ }^{3}$ Department of Electrical Engineering, Kermanshah University of Technology, \\ Kermanshah, Iran
}

\begin{abstract}
In this work, a novel structure of a microstrip diplexer consisting of coupled patch cells is presented. It works at $2.5 \mathrm{GHz}$ and 4.7 GHz for wireless applications. The proposed structure is well miniaturized with a compact area of $0.015 \lambda_{\mathrm{g}}{ }^{2}$, fabricated on $\mathbf{0 . 7 8 7} \mathbf{~ m m}$ substrate height. It has two wide fractional bandwidths (FBWs) of $28 \%$ and $17.9 \%$ at the lower and upper channels, respectively. Another feature of the proposed design is the low group delays, which are better than $0.4 \mathrm{~ns}$ for both channels. Moreover, the designed diplexer can suppress the harmonics up to $10 \mathrm{GHz}$. Meanwhile, the insertion losses at both channels are low. The design method is based on proposing an approximated equivalent $L C$ circuit of a novel basic resonator. The information about the resonator behavior is extracted from the even and odd modes analysis of the proposed equivalent $L C$ circuit. Finally, our introduced diplexer is fabricated and measured to verify the simulation results, where the simulated and measured results are in a good agreement.
\end{abstract}

Index Terms - Fractional bandwidth, Group delay, Harmonics, Microstrip diplexer, Wireless applications.

\section{INTRODUCTION}

Microstrip diplexers are attractive devices for the frequency domain multiplexing in modern communication systems (Yahya, Rezaei and Nouri, 2020a). Several types of microstrip structures have been used to design diplexers for wireless applications (Rezaei, Yahya and Jamaluddin, 2020; Rezaei, Nouri and Mohammadi, 2019; Huang, et al., 2016; Rezaei, et al., 2019a; Jun-Mei, Zhou and Cao, 2016; Peng

ARO-The Scientific Journal of Koya University Vol. VIII, No.2 (2020), Article ID: ARO.10753, 7 pages DOI:10.14500/aro.10753

Received: 12 September 2019; Accepted: 18 November 2020

Regular research paper: Published: 17 December 2020

Correspondent author's e-mail: salah.ismaeel@koyauniversity.org

Copyright (C) 2020 Salah I. Yahya and Abbas Rezaei. This is an openaccess article distributed under the Creative Commons Attribution License. and Chiang, 2015; Rezaei, et al., 2019b; Salehi, et al., 2016; Rezaei and Noori, 2018; Chen, 2015; Xiao, 2015; Noori and Rezaei, 2017a; Rezaei, Noori and Mohamadi, 2017, Noori and Rezaei, 2017b; Bui, et al., 2017; Guan, et al., 2014; Bukuru, Song and Xue, 2015; Yahya, Rezaei and Nouri, 2020b; Rezaei, et al., 2020). However, all of these diplexers occupy a large implementation area. Meanwhile, they do not have wide fractional bandwidths (FBWs) for broadband applications.

In (Rezaei, Yahya and Jamaluddin, 2020), engraved patch cells divided into trapezius and triangle cells have been utilized to design a microstrip diplexer. This structure is suitable for harmonic suppression with several transmission zeros (TZs) at its stopband. Two meandrous cells have been coupled to design a microstrip diplexer in (Rezaei, Nouri and Mohammadi, 2019). This diplexer could not suppress the harmonics whereas it has low frequency selectivity. In (Huang, et al., 2016), coupled stub loaded U-shape cells, in (Rezaei, et al., 2019a), coupled lines loaded by similar patch cells, in (Jun-Mei, Zhou and Cao, 2016), coupled U-shape structure, in (Peng and Chiang, 2015), meandrous close-loops connected to interdigital cells, in (Rezaei, et al., 2019b), coupled meandrous cells and in (Salehi, et al., 2016), and triangular open-loop resonators have been used to obtain dual-band band pass-band pass diplexers.

The above-mentioned diplexers could not attenuate the $4^{\text {th }}$ harmonics except the introduced diplexer in (Salehi, et al., 2016), which could suppress the harmonics from $1^{\text {st }}$ up to $4^{\text {th }}$ harmonics. Similarly, the other designed diplexers (Rezaei and Noori, 2018; Chen, 2015; Xiao, 2015; Noori and Rezaei, 2017a; Rezaei, Noori and Mohamadi, 2017, Noori and Rezaei, 2017b; Bui, et al., 2017; Guan, et al., 2014; Bukuru, Song and Xue, 2015; Yahya) have the problem of unsuppressed harmonics. The proposed diplexer in Rezaei and Noori, 2018, which has been proposed based on novel microstrip engraved patch cells, has low insertion losses at both channels and several TZs at its stopband. Slotlineloaded microstrip ring resonators (Chen, 2015), open-loops 
connected by mixed electromagnetic coupling structure (Xiao 2015), coupled stub loaded microstrip lines (Noori and Rezaei, 2017a) and coupled meandrous cells (Rezaei, Noori and Mohamadi, 2017) have been used to design new microstrip diplexers.

The reported diplexers in (Chen, 2015; Xiao, 2015), occupy large areas with high insertion losses at upper and lower channels. As mentioned above, some of the reported diplexers have similar resonators. However, the designed diplexer in Noori and Rezaei, 2017b, has a novel structure. Nevertheless, the isolation between channels of this diplexer is low. Interdigital cells in (Bui, et al., 2017), two coupled E-shape structures in (Guan, et al., 2014), and coupled spiral resonators in (Bukuru, Song and Xue, 2015), have been used to design microstrip diplexers. In (Yahya, Rezaei and Nouri, 2020b), a high-performance microstrip multiplexer has been designed using computational intelligence for multi-band RF wireless communications systems. In (Rezaei, et al., 2020), a low-loss compact microstrip diplexer has been designed based on coupled meandrous open-loop resonators.

Since the diplexers with compact area, low group delay and wide FBWs are rarely designed, in this work we have designed a compact microstrip diplexer with wide FBWs and very low group delay for wireless applications. Moreover, our diplexer can attenuate from the $1^{\text {st }}$ to $4^{\text {th }}$ harmonics whereas it has low insertion losses at both channels, several TZs at its stopband and acceptable isolation and return loss. The designing method is based on proposing a symmetric singlemode resonator without harmonics. Then, the odd and even modes analysis is carried out to determine the microstrip cells with the most impact on the resonator behavior. Using the proposed designing method, we can miniaturize the area. Meanwhile, we show that our resonator can suppress the harmonics inherently.

\section{Design and Analysis of the Proposed Structure}

The proposed resonator is composed microstrip thin sections, patch cells, and coupling structure. The coupling structure creates small capacitors named coupling capacitors whereas the thin cells have inductance features. The simulation results show that using the patch cells can help to save the area. Fig. 1a shows the proposed resonator. It includes two coupled sections with a symmetric structure. As depicted in Fig. 1a, in each section there are two rectangular cells that are coupled to each other. These cells create two capacitors larger than the coupling and gap capacitors.

An approximated equivalent $L C$ circuit of the proposed resonator is illustrated in Fig. 1b. The stubs with the physical lengths $l_{1}, l_{2}$, and $l_{3}$ are replaced with the inductors $L_{1}, L_{2}$, and $L_{3}$, respectively. The coupling, gap, and rectangular capacitors are $C_{\mathrm{C}}, C_{\mathrm{g}}$, and $C_{\mathrm{r}}$, respectively. In the approximated $L C$ model, the effects of bents and steps in widths are removed, as they are important only at the frequencies higher than $10 \mathrm{GHz}$ (Jahanbakhshi and Hayati, 2016). Moreover, replacing the coupling effect by only three capacitors is an approximated model for the coupling structure because in the exact model the number of capacitors will be increased significantly (Rezaei and Noori, 2018).

To obtain some information about the resonator behavior, we analyzed the $L C$ model. The impedance between the input and output ports $(Z)$ is calculated as follows:

$$
\begin{aligned}
& Z= \frac{\left(2 j \omega L_{1}+Z_{A}\right) \times\left(2 j \omega L_{3}+\frac{1}{j \omega C_{g}}\right)}{\left(2 j \omega L_{1}+Z_{A}\right)+\left(2 j \omega L_{3}+\frac{1}{j \omega C_{g}}\right)} \\
&\left.\left(\frac{1}{j \omega C_{C}}+\frac{2}{j \omega C_{r}}\right) \times \frac{1}{j \omega C_{C}}+2 j \omega L_{2}\right) \times \frac{1}{j \omega C_{C}} \\
& \text { where: } \begin{aligned}
\left(\frac{1}{j \omega C_{C}}+\frac{2}{j \omega C_{r}}\right)+\frac{1}{j \omega C_{C}} \\
\quad
\end{aligned} \\
& \frac{\left(\frac{1}{j \omega C_{C}}+\frac{2}{j \omega C_{r}}\right) \times \frac{1}{j \omega C_{C}}}{\left(\frac{1}{j \omega C_{C}}+\frac{2}{j \omega C_{r}}\right)+\frac{1}{j \omega C_{C}}}+2 j \omega L_{2}+\frac{1}{j \omega C_{C}} \\
& Z_{A}= j \frac{4 \omega^{2} L_{2}\left(1+\frac{C_{C}}{C_{r}}\right)-\frac{1}{C_{C}}-\frac{2}{C_{r}}}{\omega\left(3+\frac{4 C_{C}}{C_{r}}-4 \omega^{2} L_{2} C_{C}\left(1+\frac{C_{C}}{C_{r}}\right)\right)}
\end{aligned}
$$

In (1), $\omega$ is an angular frequency. The coupling capacitor is a small value in $\mathrm{fF}$. Therefore, for $\mathrm{C}_{\mathrm{c}}<<\mathrm{C}_{\mathrm{r}}$ we can write $1+C_{C} / C_{r} \approx 1$ and $3+4 C_{C} / C_{r} \approx 3$. As a result, $\mathrm{ZA}$ and $\mathrm{Z}$ will be changed as follows:

$$
\begin{aligned}
& Z_{A}= j \frac{4 \omega^{2} L_{2}-\frac{1}{C_{C}}-\frac{2}{C_{r}}}{\omega\left(3-4 \omega^{2} L_{2} C_{C}\right)} \Rightarrow \\
& Z=j \frac{\left(\omega^{2}\left(6 L_{1}+4 L_{2}\right)-8 \omega^{4} L_{2} C_{C} L_{1}-\frac{1}{C_{C}}-\frac{2}{C_{r}}\right)}{\omega\left(1-2 \omega^{2} L_{3} C_{g}\right)} \\
&+\left[3+\frac{C_{g}}{C_{C}}+\frac{2 C_{g}}{C_{r}}-\omega^{2}\left(4 L_{2}\left(C_{C}+C_{g}\right)+6\left(L_{1}+L_{3}\right) C_{g}\right)\right. \\
&\left.+8 \omega^{4}\left(L_{1}+L_{3}\right) L_{2} C_{g} C_{C}\right]
\end{aligned}
$$

Similar to the coupling capacitor, the gap capacitor $\left(C_{\mathrm{g}}\right)$ is very small. Accordingly, $Z$ will be approximated as follows:

$$
Z=j \frac{\omega^{2}\left(6 L_{1}+4 L_{2}\right)-8 \omega^{4} L_{2} C_{C} L_{1}-\frac{1}{C_{C}}-\frac{2}{C_{r}}}{\omega\left[3+\frac{C_{g}}{C_{C}}\right]}
$$

For the small values of $C_{\mathrm{C}}$, we can remove the second phrase $\left(8 \omega^{4} L_{2} C_{C} L_{1}\right)$ in the numerator of (3), so that:

$$
Z=j \frac{\omega^{2}\left(6 L_{1}+4 L_{2}\right)-\left(\frac{1}{C_{C}}+\frac{2}{C_{r}}\right)}{\omega\left[3+\frac{C_{g}}{C_{C}}\right]}
$$

Due to the symmetry in the proposed resonator structure, we can carry out the even and odd modes analysis. To find the 

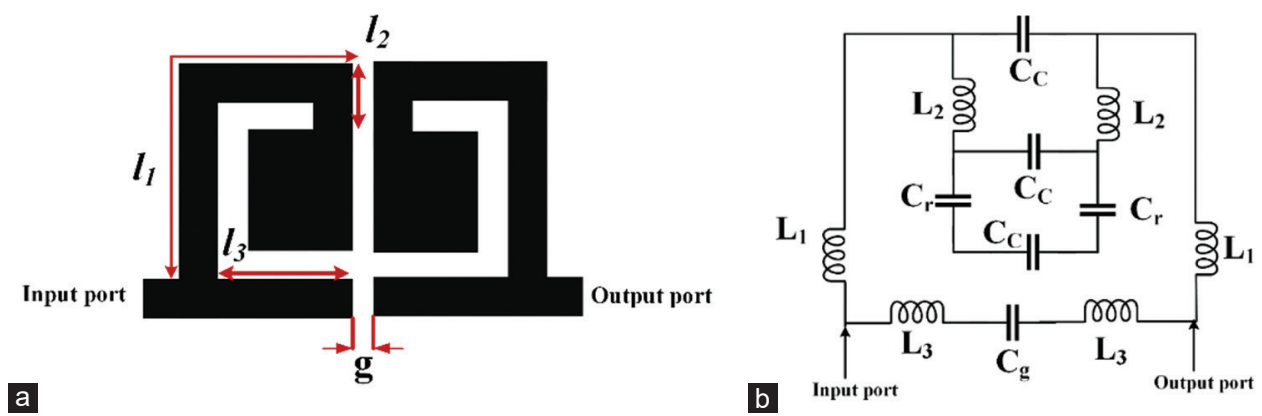

Fig. 1. (a) Layout of the proposed resonator, and (b) an equivalent $L C$ circuit of the proposed resonator.

odd mode angular resonance frequency $\left(\omega_{\mathrm{n}}\right)$, the numerator of the above equation must be zero. On the other hand, for calculating the even mode angular resonance frequency the denominator of the above equation should be zero. Hence, we have only an odd mode angular resonance frequency as follows:

$$
\omega_{o}=\sqrt{\frac{C_{r}+2 C_{C}}{2 C_{C} C_{r}\left(3 L_{1}+2 L_{2}\right)}}
$$

Since we have only an odd mode resonance frequency, our proposed resonator is a single-mode resonator with the attenuated harmonics. Because, if we had more resonance frequencies they were harmonics. If we apply the approximation of the small coupling capacitor, (4) can be decreased as follows:

$$
\omega_{o}=\sqrt{\frac{1}{2 C_{C}\left(3 L_{1}+2 L_{2}\right)}}
$$

According to (6), we can tune the resonance frequency by selecting the appropriate values of the inductors $L_{1}$, $L_{2}$ and a coupling capacitor. Therefore, the resonance frequency depends on the space between the coupled cells and the physical lengths $l_{1}$ and $l_{2}$, significantly. Hence, for a predetermined angular resonance frequency, we can select the space between the coupled sections and dimensions of the physical lengths $l_{1}$ and $l_{2}$ in a way that we can save the area.

If the gap between the coupled sections can be reduced, the coupling capacitor will be increased. As a result, for a constant predetermined angular resonance frequency, we can decrease the dimensions of the physical lengths $l_{1}$ and $l_{2}$ which leads to save the area. Extracting the above information about the resonator behavior leads to better optimization, easily.

By knowing the basic information, we can design two band pass filters (BPFs) (BPF1 and BPF2) with compact size and attenuated harmonics as depicted in Fig. 2a and b, respectively. In Fig. 2a and b, the dimensions of both filters are in $\mathrm{mm}$. To design these BPFs, the proposed resonator is utilized. However, for BPF1 the space between cells is smaller whereas it has extra stubs that are used for better optimization. To improve the insertion losses, the patch rectangular feed structures are added to both BPFs. The bandwidths of BPF1 and BPF2 are affected by their overall sizes. Increasing the overall size of each BPF decreases the bandwidth, whereas the fractional bandwidth (FBW) remains constant.

The frequency responses of BPF1 and BPF2 are depicted in Fig. $2 c$ and d, respectively. The simulation results are obtained using the EM simulator of ADS software on a Rogers RT/duroid 5880 substrate with $\varepsilon_{\mathrm{r}}=2.2$, loss tangent $=0.0009$, and the substrate thickness $0.787 \mathrm{~mm}$. As shown in Fig. 2, BPF1 works at $2.49 \mathrm{GHz}$ whereas it has a very low simulated insertion loss of $0.02 \mathrm{~dB}$ and a wide FBW of $30 \%$. It suppresses the harmonics up to $9.9 \mathrm{GHz}$ with the maximum harmonic level $20 \mathrm{~dB}$. Furthermore, BPF2 operates at $4.9 \mathrm{GHz}$ with $0.7 \mathrm{~dB}$ insertion loss and an FBW of $28.7 \%$. It suppresses the harmonics up to $18.6 \mathrm{GHz}$ with the maximum harmonic level $20 \mathrm{~dB}$.

To obtain a dual-band band pass-band pass diplexer, we connected BPF1 and BPF2 with the same dimensions given in Fig. $2 \mathrm{a}$ and b. Fig. 3 illustrates the layout configuration of the proposed diplexer. As shown in Fig. 3, there is no need to use an extra junction for connecting the BPFs and this can save the area. This is due to having several coupling structures between Ports 2 and 3, whereas there are not any paths without coupling between Ports 2 and 3. Therefore, the BPFs do not have a significant loading effect on each other.

\section{Results AND Discussion}

We simulated the proposed diplexer using the EM simulator of Advanced Design System (ADS) software. The designed diplexer is fabricated on a Rogers RT/duroid 5880 substrate with $\varepsilon_{\mathrm{r}}=2.22$, loss tangent $=0.0009$, and the substrate thickness $0.7874 \mathrm{~mm}$. We used Agilent network analyzer $\mathrm{N} 5230 \mathrm{~A}$ to obtain the measurement results of the fabricated diplexer. The proposed diplexer occupies a very small area of $13.3 \mathrm{~mm} \times 10.2 \mathrm{~mm}=0.14 \lambda_{\mathrm{g}} \times 0.11 \lambda_{\mathrm{g}}$, where $\lambda_{\mathrm{g}}$ is the guided wavelength calculated at the first resonance frequency.

Fig. 4a shows the simulated and measured $S_{21}$ and $S_{31}$. As depicted in Fig. 4a, the designed diplexer works at $f_{1}=2.5$ $\mathrm{GHz}$ and $f_{2}=4.7 \mathrm{GHz}$ with two low insertion losses better than $0.2 \mathrm{~dB}$ at both channels. However, due to copper and junction losses, the measured losses are a little higher than the simulated losses. The first and second FBWs are $28 \%$ and $17.9 \%$, respectively. As shown in Fig. $4 \mathrm{a}$, the designed diplexer can attenuate the harmonics up to $10 \mathrm{GHz}\left(4 f_{1}\right)$ with the maximum harmonic level $16.2 \mathrm{~dB}$. Therefore, it can attenuate $1^{\text {st }}, 2^{\text {nd }}, 3^{\text {rd }}$, and $4^{\text {th }}$ harmonics.

Fig. $4 \mathrm{~b}$ and $\mathrm{c}$ illustrate the simulated and measured isolation between channels and common port return loss, respectively. The common port return losses at the lower 

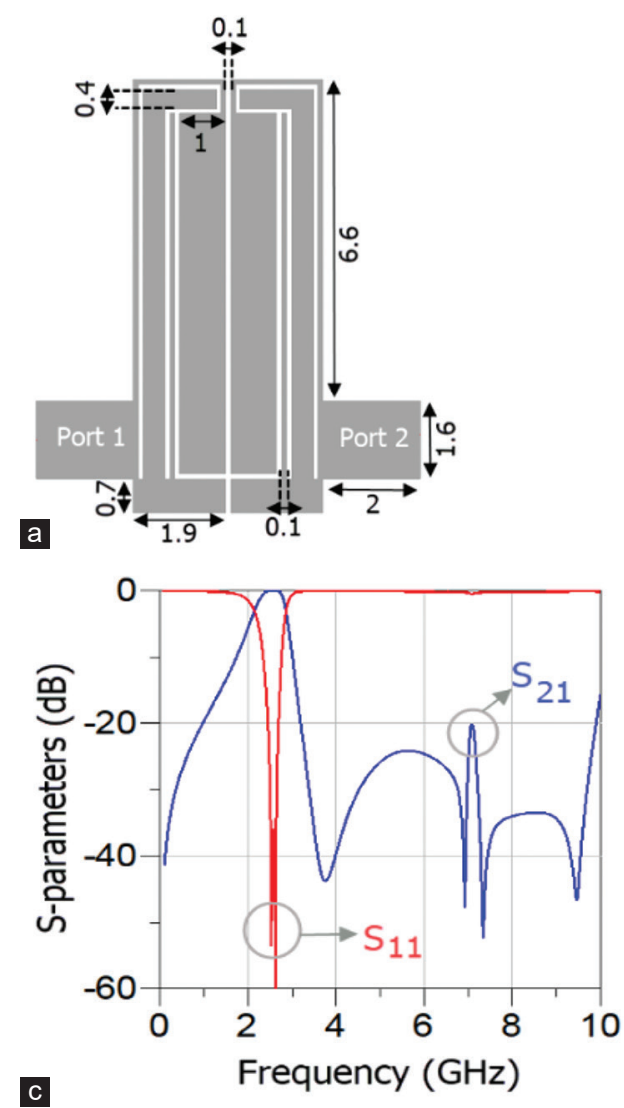

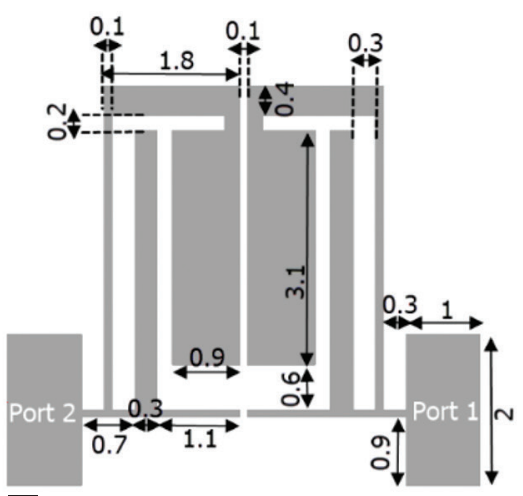

b

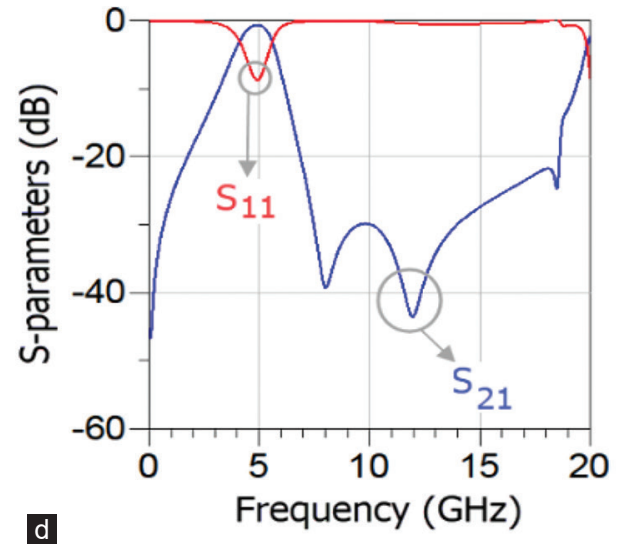

Fig. 2. (a) Layout of BPF1, (b) layout of BPF2, (c) frequency response of BPF1, and (d) frequency response of BPF2.

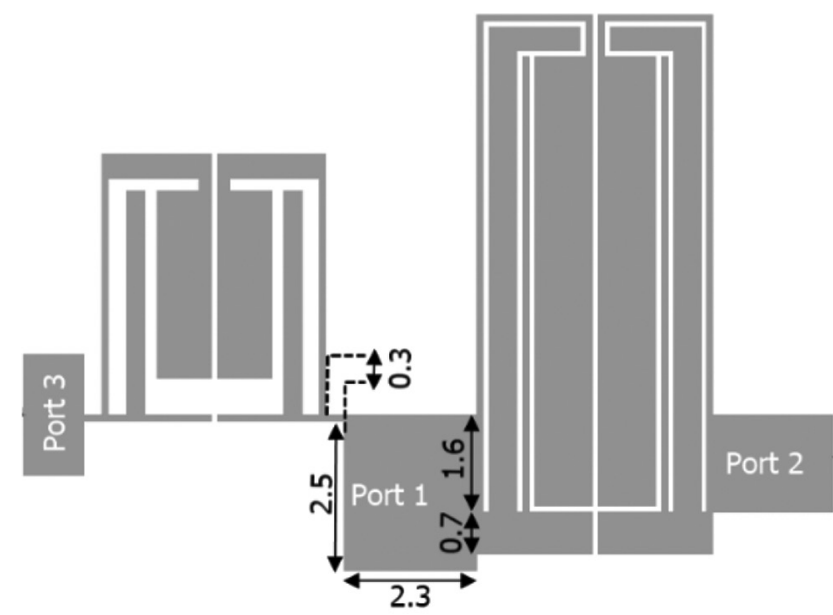

Fig. 3. Layout configuration of our diplexer (all dimensions are in $\mathrm{mm}$ ).

and upper channels are better than $15.9 \mathrm{~dB}$ and $17 \mathrm{~dB}$, respectively, whereas there are two transmission poles at the first channel. Meanwhile, the reasonable isolation between the channels better than $20 \mathrm{~dB}$ is obtained. A photograph of the fabricated diplexer is presented in Fig. 4c.

To prove the advantages of this work, we compared our diplexer with the previously reported microstrip diplexers, as shown in Table I. From Table I it can be seen that, in comparison with the previously reported diplexers, the proposed diplexer in this work has the most compact area and the widest FBWs, whereas it has low insertion losses at both channels. Moreover, it can attenuate the harmonics better than all; expect the introduced diplexers in (Rezaei, Yahya and Jamaluddin, 2020; Salehi, et al., 2016). However, these two reported diplexers occupy larger areas. Meanwhile, the channels of these diplexers are narrower than ours.

In addition to the mentioned features, the proposed diplexer has flat channels with low group delays. Fig. 5a and b show the group delays of the first and second channels, respectively. The narrowband transmission parameters of the proposed diplexer are depicted in Fig. 5c and d. The simulated group delays of the proposed diplexer are lower than 0.4 ns which are very small value for a microstrip diplexer. Despite the importance of having flat channels (a non-flat pass band with high group delay has a pulse distortion), most of the previously reported diplexers did not pay attention to this problem. Therefore, we had to compare the group delay of our work with some of the previously reported diplexers and filters, as shown in Table II. In comparison with the previous works, our diplexer has the minimum group delays at both channels.

The isolation of the proposed diplexer is a function of the gap between the first and second channels, which is depicted in Fig. 6. Using a smaller filter shifts the frequency response to the right. Accordingly to change the gap between the channels, we changed the overall dimension of the larger filter with the scales $1: 1,0.9: 1,0.8: 1$, and $0.7: 1$ so that the gap between channels are obtained $2.25 \mathrm{GHz}, 2.22 \mathrm{GHz}$, $2.1 \mathrm{GHz}$, and $1.33 \mathrm{GHz}$, respectively. As shown in Fig. 6, by increasing the gap between channels, the isolation of the proposed diplexer will be improved. 

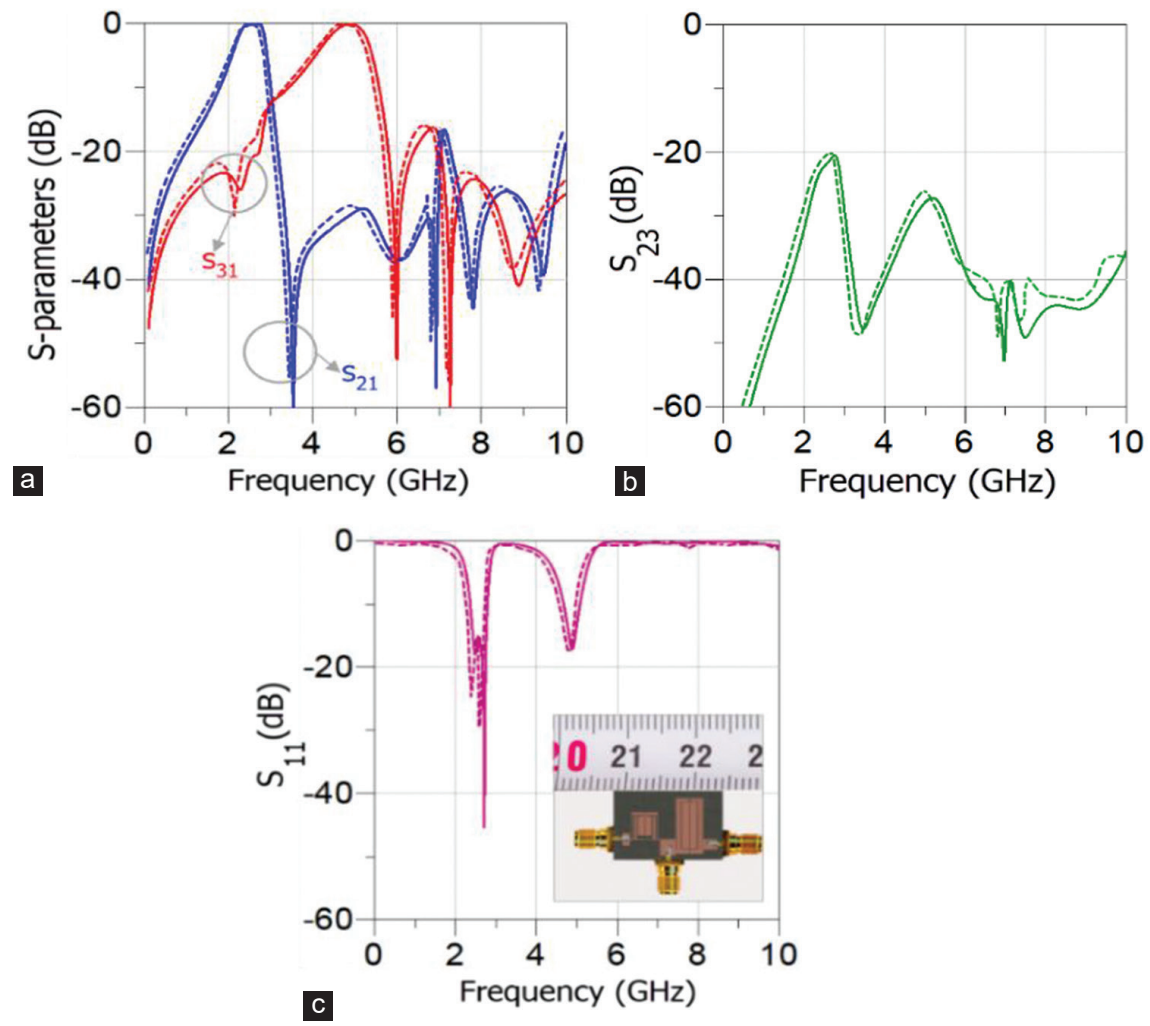

Fig. 4. (a) Simulated (solid line) and measured (dashed line) $S_{21}$ and $S_{31}$, (b) simulated (solid line) and measured (dashed line) $S_{23}$, and (c) simulated (solid line) and measured (dashed line) $S_{11}$ with a photograph of the fabricated diplexer.
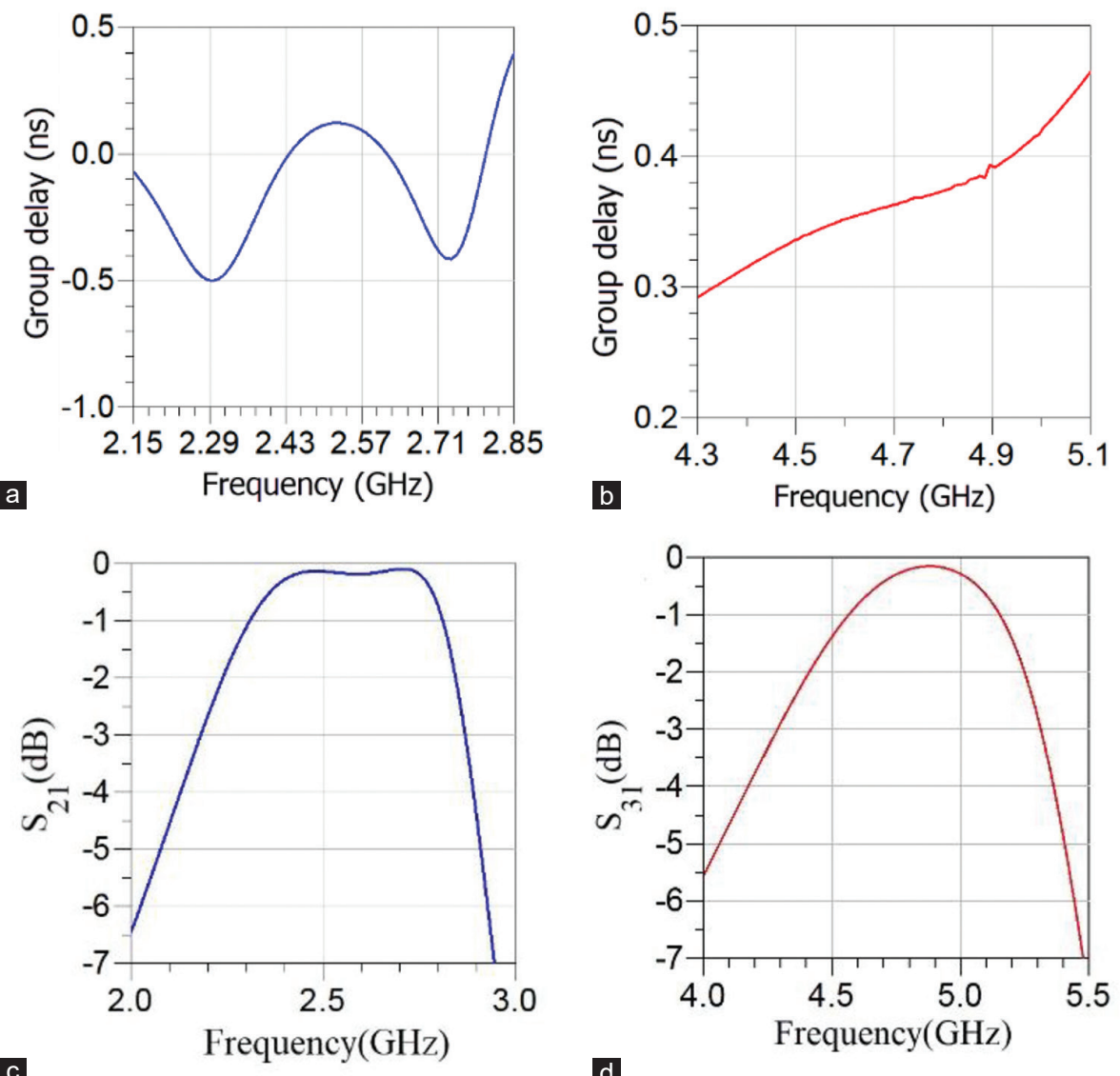

Fig. 5. (a) Simulated group delay of the first band, (b) simulated group delay of the second band (c) narrowband frequency response of $S_{21}$, (d) narrowband frequency response of $S_{31}$. 
TABLE I

Comparison Between this Work and the Previously Reported Microstrip Diplexers

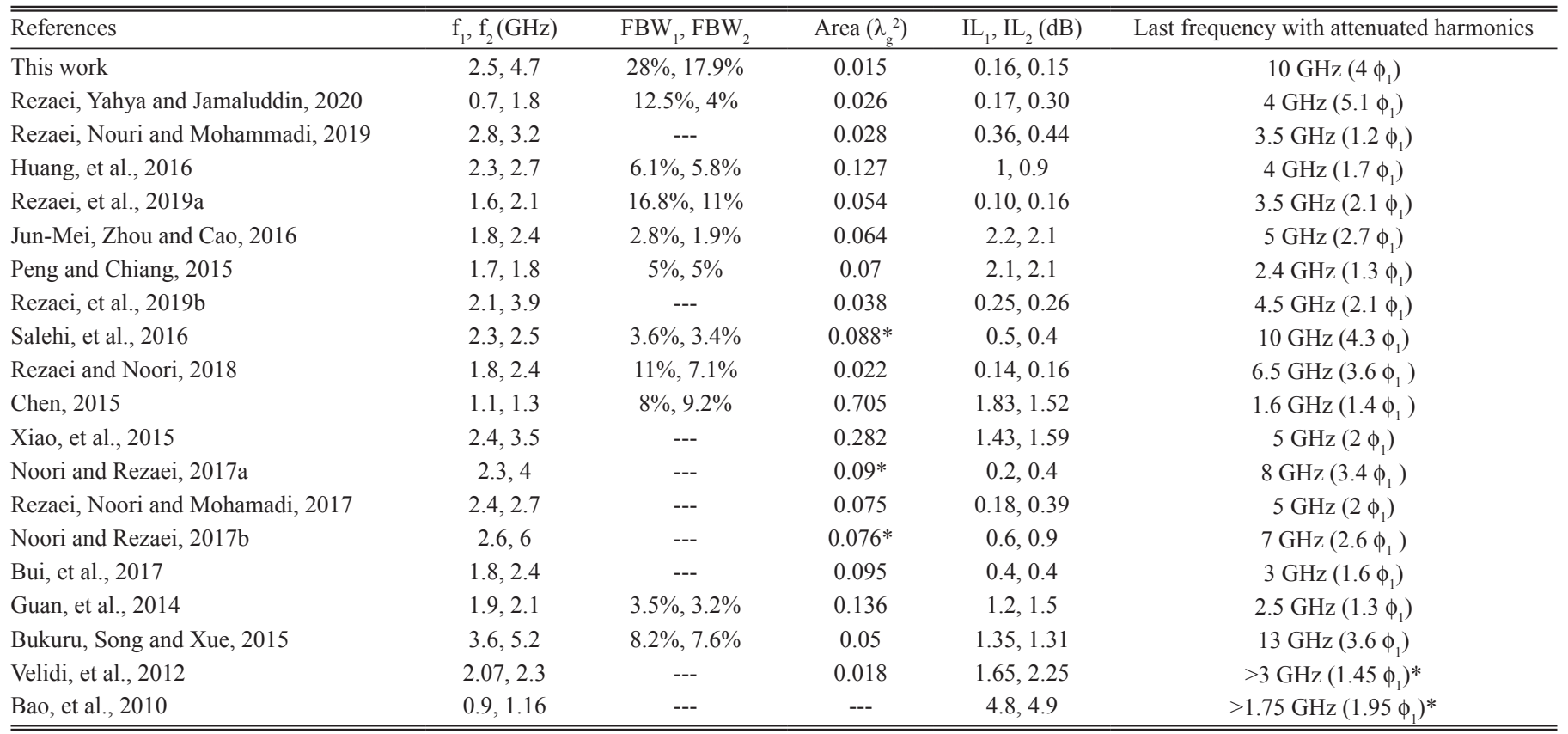

*Approximated values

TABLE II

The Maximum Group Delay of this Work in Comparison with the Previously Reported Microstrip Diplexers and Filters

\begin{tabular}{lccc}
\hline \hline References & Type & $\begin{array}{c}\text { Number of } \\
\text { channels }\end{array}$ & $\begin{array}{c}\text { Maximum group } \\
\text { delay (ns) }\end{array}$ \\
\hline This work & Diplexer & 2 & 0.4 \\
Rezaei, et al., 2019a & Diplexer & 2 & 2.6 \\
Rezaei, et al., 2019b & Diplexer & 2 & 4 \\
Rezaei and Noori, 2018 & Diplexer & 2 & 3.14 \\
Noori and Rezaei, 2017b & Diplexer & 2 & 3 \\
Sarkar, Ghatak and Poddar, 2011 Filter & 2 & 2.5 \\
Liu, 2010 & Filter & 3 & 8 \\
Wibisono, Firmansyah and & Filter & 3 & 3.67 \\
Syaфraditya, 2016 & & & \\
Lin, 2011 & Filter & 4 & 8 \\
\hline \hline
\end{tabular}

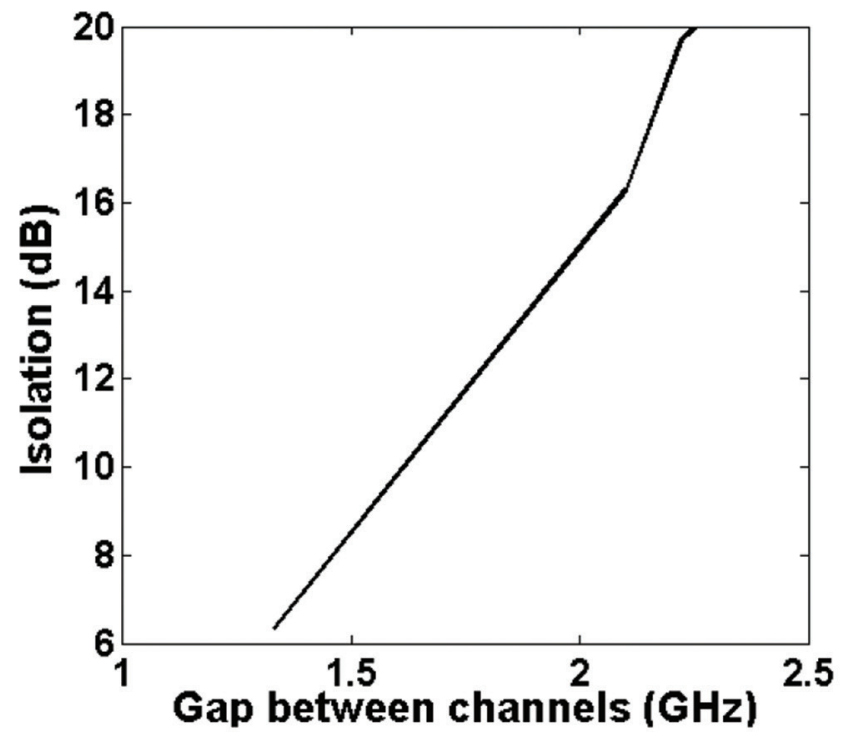

Fig. 6. Isolation of the proposed diplexer as a function of the channels spacing.

\section{CONCLUSION}

A microstrip diplexer with compact size, wide fractional bandwidths (FBWs), low group delays, low insertion losses, and attenuated harmonics was designed in this work for wireless applications. Using the even and odd modes analysis of an introduced equivalent $L C$ circuit, the significant microstrip sections that affect the frequency response of the proposed resonator were identified. As a result, for a predetermined angular resonance frequency, we could select the dimensions and space between the coupled sections. We obtained some information about the behavior of the introduced resonator by analyzing the proposed equivalent $L C$ circuit. This information led to better optimization so that we could decrease the overall size of the proposed structure. Therefore, the proposed diplexer has a very compact area of $0.015 \lambda_{\mathrm{g}}{ }^{2}$. By tuning the dimensions of the feed structures, we could improve the insertion losses, where they are better than $0.2 \mathrm{~dB}$ at both channels. These features were obtained whereas the isolation between channels and return losses at both channels are acceptable.

\section{REFERENCES}

Bao, Z., Chen, J., Lim, E.H. and Xue, Q., 2010. Compact microstrip diplexer with differential outputs. Electronics Letters, 46(11), pp.766-768.

Bui, D.H.N., Vuong, T.P., Allard, B., Verdier, J. and Benech, P., 2017. Compact low-loss microstrip diplexer for RF energy harvesting. IET Electronic Letters, 53(8), pp.552-554.

Bukuru, D., Song, K. and Xue, Q., 2015. Compact wide-stopband planar diplexer based on rectangular dual spiral resonator. Microwave and Optical Technology Letters, 57(1), pp.174-178.

Chen, D., Zhu, L., Bu, H. and Cheng, C.H., 2015. A novel planar diplexer using slot line-loaded microstrip ring resonator. IEEE Microwave and Wireless Components Letters, 25(11), pp.706-708. 
Guan, X., Yang, F., Liu, H. and Zhu, L., 2014. Compact and high-isolation diplexer using dual-mode stub-loaded resonators. IEEE Microwave and Wireless Components Letters, 24(6), pp.385-387.

Huang, F., Wang, J., Zhu, L. and Wu, W., 2016. Compact microstrip balun diplexer using stub-loaded dual-mode resonators. IET Electronic Letters, 52, pp.1994-1996.

Jahanbakhshi, M. and Hayati, M., 2016. Design of a compact microstrip lowpass filter with sharp roll-off using combined T-shaped and L-shaped resonators. IET Electronic Letters, 52(23), pp.1931-1933.

Jun-Mei, Y., Zhou, H.Y. and Cao, L.Z., 2016. Compact diplexer using microstrip half- and quarter wavelength resonators. IET Electronic Letters, 52(19), pp.1613-1615.

Lin, S.C., 2011. Microstrip dual/quad-band filters with coupled lines and quasi-lumped impedance inverters based on parallel path transmission. IEEE Transactions on Microwave Theory and Techniques, 59(8), pp.1937-1946.

Liu, Y., 2010. A tri-band bandpass filter realized using tri-mode T-shape branches. Progress in Electromagnetics Research, 105, pp.425-444.

Noori, L. and Rezaei, A., 2017a. Design of a microstrip dual-frequency diplexer using microstrip cells analysis and coupled lines components. International Journal of Microwave and Wireless Technologies, 9(7), pp.1467-1471.

Noori, L. and Rezaei, A., 2017b. Design of a microstrip diplexer with a novel structure for WiMAX and wireless applications. AEU-International Journal of Electronics and Communications, 77, pp.18-22.

Peng, H. and Chiang, Y., 2015. Microstrip diplexer constructed with new types of dual-mode ring filters. IEEE Microwave and Wireless Components Letters, 25(1), pp.7-9.

Rezaei, A. and Noori, L., 2018. Compact low-loss microstrip diplexer using novel engraved semi-patch cells for GSM and WLAN applications. AEU-International Journal of Electronics and Communications, 87, pp.158-163.

Rezaei, A., Noori, L. and Mohamadi, H., 2017. Design of a novel compact microstrip diplexer with low insertion loss. Microwave and Optical Technology Letters, 59(7), pp.1672-1676.

Rezaei, A., Nouri, L. and Mohammadi, H., 2019. Design of a miniaturized microstrip diplexer using coupled lines and spiral structures for wireless and WiMAX applications. Analog Integrated Circuits and Signal Processing, 98, pp.409-415.

Rezaei, A., Yahya, S.I. and Jamaluddin, M.H., 2020. Anovel microstrip diplexer with compact size and high isolation for GSM applications. AEU-International Journal of Electronics and Communications, 114, p.153018.

Rezaei, A., Yahya, S.I., Noori, L. and Jamaluddin, M.H., 2019a. Design of a novel wideband microstrip diplexer using artificial neural network. Analog Integrated Circuits and Signal Processing, 101(1), pp.57-66.

Rezaei, A., Yahya, S.I., Noori, L. and Jamaluddin, M.H., 2019b. Design and fabrication of a novel compact low-loss microstrip diplexer for WCDMA and WiMAX applications. Journal of Microwaves Optoelectronics and Electromagnetic Applications, 18(4), pp.482-491.

Rezaei, A., Yahya, S.I., Nouri, L. and Jamaluddin, M.H., 2020. Design of a low-loss microstrip diplexer with a compact size based on coupled meandrous open-loop resonators. Analog Integrated Circuits and Signal Processing, 102, pp.579-584.

Sarkar, P., Ghatak, R. and Poddar, D.R., 2010. A dual-band bandpass filter using SIR suitable for WiMAX band. Vol. 6, In: 2011 Proceedings of the International Conference on Information and Electronics Engineering, pp.70-74.

Salehi, M.R., Keyvan, S., Abiri, E. and Noori, L., 2016. Compact microstrip diplexer using new design of triangular open loop resonator for $4 \mathrm{G}$ wireless communication systems. AEU-International Journal of Electronics and Communications, 70(7), pp.961-969.

Velidi, V.K., Prabhakaran, U., Subramanyam, A.V.G., Sivareddy, D. and Srinivasan, V.V., 2012. Design of compact microstrip diplexer with high selectivity. In: 2012 Proceedings of the International Conference on Signal Processing and Communications, Bangalore, pp.1-4.

Wibisono, G., Firmansyah, T. and Syafraditya, T., 2016. Design of triple-band bandpass filter using cascade tri-section stepped impedance resonators. Journal of ICT Research and Applications, 10(1), pp.43-56.

Xiao, J.K., Zhu, M., Li, Y., Tian, L. and Ma, J.G., 2015. High selective microstrip bandpass filter and diplexer with mixed electromagnetic coupling. IEEE Microwave and Wireless Components Letters, 25(12), pp.781-783.

Yahya, S.I., Rezaei, A. and Nouri, L., 2020a. Design and performance of microstrip diplexers: A review. ARO-The Scientific Journal of Koya University, 8(1), pp.38-49.

Yahya, S.I., Rezaei, A. and Nouri, L., 2020b. Design and fabrication of a high-performance microstrip multiplexer using computational intelligence for multi-band RF wireless communications systems. AEU-International Journal of Electronics and Communications, 120, p.153190. 